\title{
马兰戈尼效应在有机半导体成膜中的应用
}

吴凯

北京大学化学与分子工程学院, 北京 100871

\section{Application of Marangoni Effect in the Fabrication of Organic Semiconductor Films}

WU Kai

College of Chemistry and Molecular Engineering, Peking University, Beijing 100871, P. R. China.

Email: kaiwu@pku.edu.cn.

Published online: June 27, 2019.

\section{1 背景介绍}

以有机发光二极管、有机太阳能电池和有机 场效应晶体管为主的有机半导体薄膜器件, 因在 便携、可穿戴电子器件领域有巨大的应用前景, 而 逐渐成为无机半导体器件的一个重要补充。溶液 法生长有机半导体薄膜工艺更使有机半导体器件 的低成本、可在柔性基底上大面积制备的优点得 到体现, 因而受到广泛关注。

但是, 溶液法制备工艺也存在着诸多不足, 比 如被称为 “咖啡环” 效应的溶剂自然挥发造成的薄 膜不均匀性现象等 1 。针对薄膜的不均匀性, 出现 了表面修饰、使用混合溶剂和特殊结构等改进方 法 $^{2}$, 而在成膜溶液中构筑马兰戈尼流就是其中的 一种 ${ }^{3}$ 。

清华大学化学系董桂芳副教授课题组采用基 于马兰戈尼与咖啡环效应协同作用的方法, 利用浸 润提拉及氧等离子体选区处理工艺, 生长出图案化 的C8-BTBT薄膜, 通过对混合溶剂比例以及溶液浓 度等参数的优化, 得到高性能薄膜及基于此薄膜的 有机场效应晶体管。该工作已在物理化学学报上在 线发表(doi: 10.3866/PKU.WHXB201901056) ${ }^{4}$ 。

\section{2 研究亮点}

(1)在提拉法中, 利用具有高沸点、低表面张 力的甲苯与具有低沸点、高表面张力的四氯化碳 两种溶剂, 按比例混合, 在成膜溶液中形成马兰戈 尼流, 有效降低 “咖啡环” 效应, 提高薄膜均匀性。
(2)以疏水性氟化物CYTOP为基底, 氧等离子 体选区处理改变基底表面能, 在提拉法成膜的过 程中, 利用溶液重力、马兰戈尼与咖啡环效应协同 作用, 生长图案化的、有序的C8-BTBT薄膜, 并制 备了高性能场效应晶体管。

\section{3 图文解析}

要点 1 马兰戈尼效应与“咖啡环”效应在成膜中 的作用

液滴的边缘常具有 “钉扎效应”, 而溶剂在边 缘的挥发速度较快, 溶液会带着溶质C8-BTBT向 边缘输运, 导致溶质在边缘附近堆积, 形成咖啡环。

马兰戈尼效应是一种液体会由低表面张力区 域流向高表面张力区域的现象。在浸润提拉法中, 当选择高沸点低表面张力的甲苯溶剂与低沸点高 表面张力的四氯化碳的混合溶剂时，在快速挥发 的边缘，因低沸点的四氯化碳易于挥发而留下更 多的低表面张力的甲苯, 这就导致了边缘溶液张 力低于溶液内部, 产生了溶液带溶质离开边缘的 输运。这是马兰戈尼效应, 如图1所示, 它抑制咖 啡环的形成, 提高薄膜均匀性 ${ }^{5}$ 。同时, 基片垂直 提拉, 溶液自身的重力也会使液滴的边缘不再被 钉扎, 而是向下移动, 促成了 $\mathrm{C} 8$-BTBT的有序生长。

\section{要点 2 氧等离子体处理工艺的选择}

在浸润提拉过程中, 通过疏水的表面上形成 亲水的区域, 可以有效调控溶液在基片表面的流 动, 进而控制溶质成核、成膜的区域。文章中, 作 


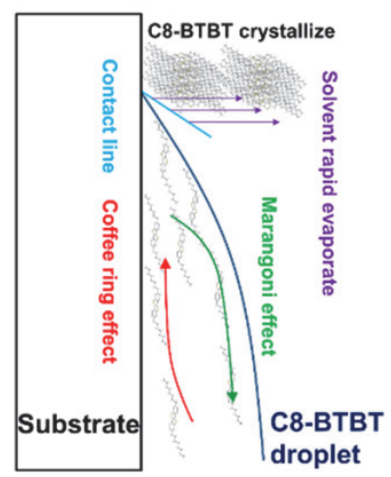

图1 马兰戈尼- “咖啡环” 效应协同作用原理示意图。

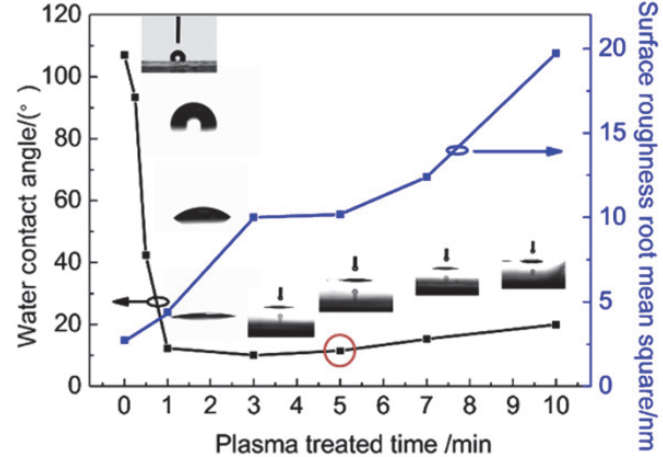

图2 水接触角和表面粗䊁度随等离子体处理时间的变化。
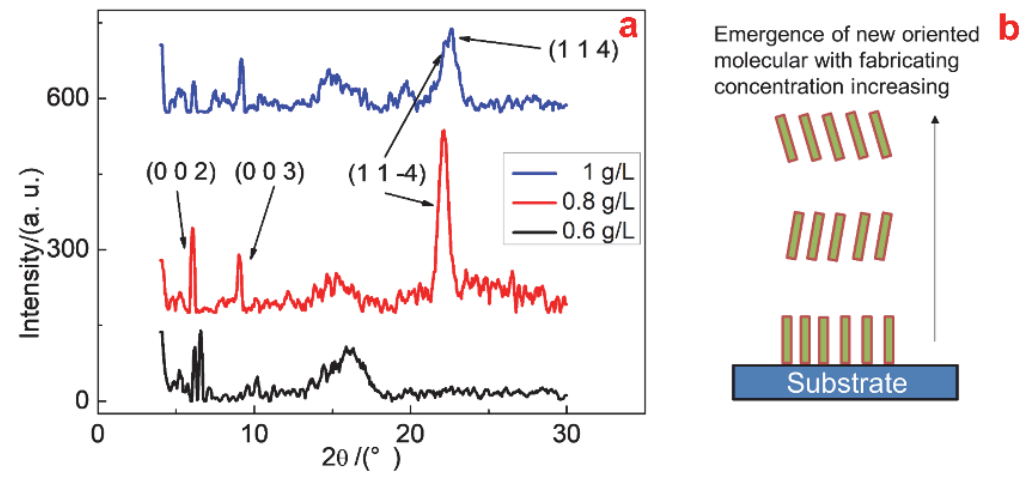

图 3 (a)不同浓度下成膜的 GIXRD 面外测试图; (b)随浓度增加出现新分子取向示意图。
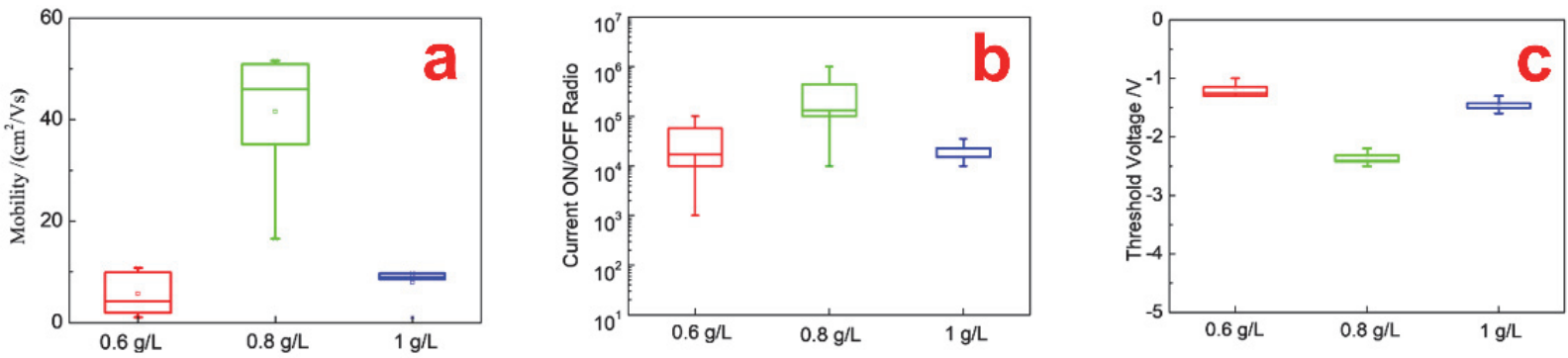

图 4 不同浓度条件下 6 个器件性能分布图: (a)迁移率, (b)开关电流比, (c)阈值电压。

者采用氧等离子体选择处理CYTOP疏水表面的方 法, 实现图案化成膜的目的。图2显示了等离子体 处理时间与表面水接触角、表面粗䊁度之间的关 系, 作者在文章中从接触角小和一致性好两个方 面考虑，分析了等离子体处理最佳时间的选择。

要点 3 溶剂比例和溶液浓度对于C8-BTBT成膜 及晶体管性能的影响

溶剂中甲苯和四氯化碳体积比例的调节将改 变选区中C8-BTBT薄膜生长的连续性。分析表明, 当甲苯和四氯化碳的体积比取 $3: 2$ 时, 薄膜的连续 性和有序性得到兼顾。C8-BTBT分子在不同成膜 条件下会出现不同的取向 ${ }^{6,7}$, 作者利用GIXRD分 析了溶液浓度分别为 $0.6 、 0.8$ 和 $1 \mathrm{~g} \mathrm{~L}^{-1}$ 条件下生长 的C8-BTBT薄膜中分子的取向特性(图3), 还讨论
了对应晶体管器件的迁移率、开关比、阈值电压大 小及分布的均匀性(图4)。

\section{4 全文小结}

该工作报道了在疏水基片上经过氧等离子体 选区处理、再利用马兰戈尼与咖啡环效应协同作 用生长图案化的 C8-BTBT半导体薄膜的思路和工 艺, 着重分析了工艺参数对 C8-BTBT薄膜及晶体 管性能的影响。该工作对溶液法制备图案化有序 薄膜工作具有一定的参考价值。

\section{References}

(1) Deegan, R. D.; Bakajin, O.; Dupont, T. F.; Huber, G.; Nagel, S. R.; Witten, T. A. Nature 1997, 389 (6653), 827. 
doi: 10.1038 /nature39827

(2) De Gan, B. J.; Schubert, U. S. Langmuir 2004, 20, 7789. doi:10.1021/la049469o

(3) Lim, J. A.; Lee, W. H.; Lee, H. S.; Lee, J. H.; Park, Y. D.; Cho, L. Adv. Funct. Mater. 2008, 18, 229. doi: 10.1002/adfm.200700859

(4) Wang, Z. A.; Guo, H.; Rong, X.; Dong, G. F. Acta Phys. -Chim. Sin. 2019, 35 (11), 1259. [王子昂, 郭航, 荣欣, 董桂芳. 物理化学学报, 2019, 35 (11), 1259.] doi: 10.3866/PKU.WHXB201901056
(5) Zhao, H.; Wang, Z.; Dong, G.; Duan, L. Phys. Chem. Chem. Phys. 2015, 17 (9), 6274. doi: 10.1039/c4cp05378h

(6) Minemawari, H.; Yamada, T.; Matsui, H.; Tsutsumi, J.; Haas, S.; Chiba, R.; Kumai, R.; Hasegawa, T. Nature 2011, 475 (7356), 364. doi: 10.1038 /nature10313

(7) Uemura, T.; Hirose, Y.; Uno, M.; Takimiya, K.; Takeya, J. Appl. Phys. Express 2009, 2, 111501.

doi: 10.1143/apex.2.111501 